SUPPORTING INFORMATION

\title{
Rational application of $\beta$-hydroxybutyrate attenuates ischemic stroke by suppressing oxidative stress and mitochondrial-dependent apoptosis via activation of the Erk/CREB/eNOS pathway
}

Yang Li, Xuepeng Zhang, Aijia Ma, Yan Kang

Intensive care unit, West China School of Medicine/West China Hospital of Sichuan University,

Chengdu, Sichuan 610041, People's Republic of China

Correspondence: Yan Kang

Intensive care unit, West China School of Medicine/West China Hospital of Sichuan University

, No.37 Guoxue Alley, Wuhou District, Chengdu, Sichuan 610041, People's Republic of China

Email kangyan@scu.edu.cn

SUPPORTING INFORMATION 


\section{Supplementary Figure $\mathbf{S 1}$}

Cortical neuronal cells were cultured and identified. (A) Cells were grown for 8 days (100x). (B) Cells were grown to log phase for 11 days (100x). (C) Immunohistochemical staining of the cells at 11 days (100x). Green: NSE, Red: DAPI.
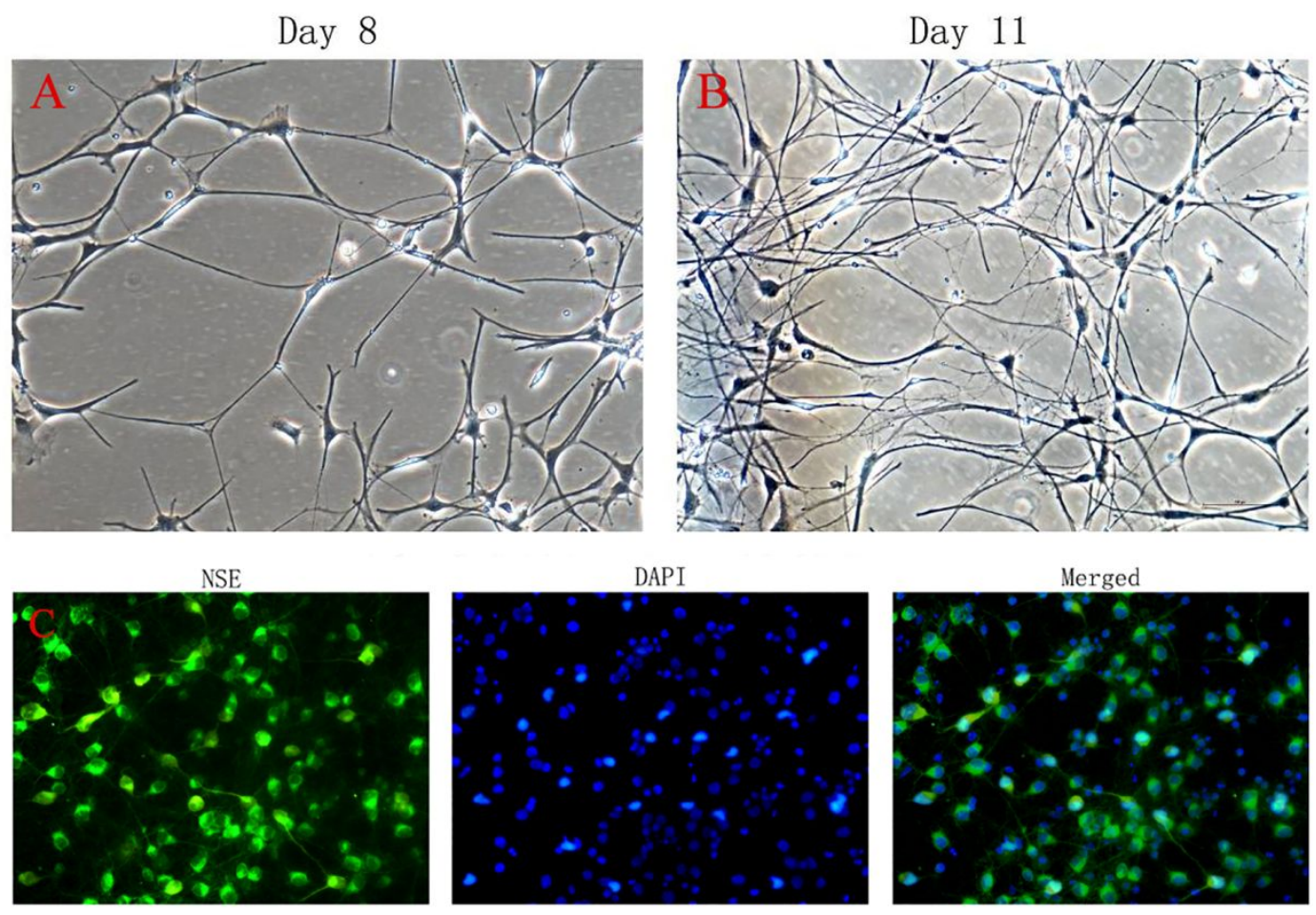


\section{Supplementary Figure S2 related to Figure 7}

In cortical neuronal cells exposed to oxygen-glucose deprivation, cells were treated with various concentrations of $\beta$-hydroxybutyrateate. $(A, B, C)$ The mRNA levels of SMCT1, Cyt-c, caspase3. Data are presented as the mean $\pm S D ; \bullet p<0.05$ vs. the Blank group; ${ }^{*} p$ $<0.05$ vs. the Hypoxia group; $\# p<0.05$ vs. the $5 \mathrm{mM}$ BHB group. $(n=5)$.

A

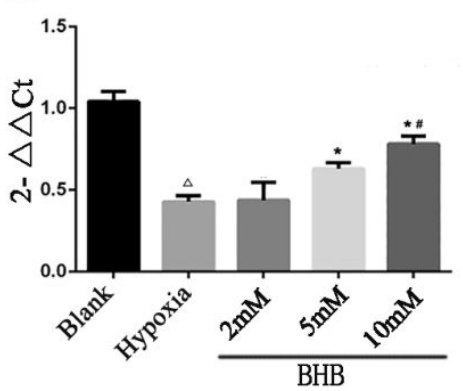

B

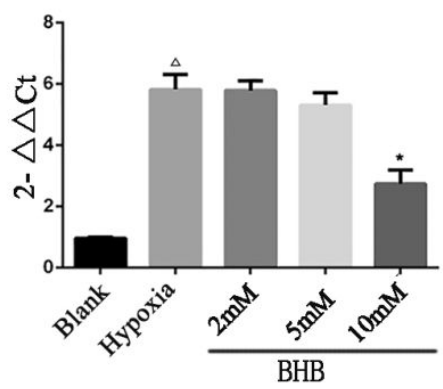

$\mathrm{C}$

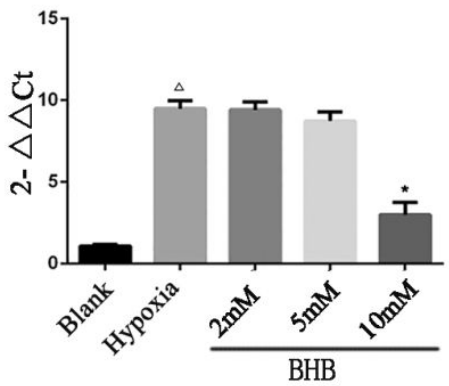

